\title{
High Dynamic Range Mapping for Synthetic Aperture Radar Images
}

\author{
Ana Gavrovska, Member, IEEE, Irini Reljin, Senior Member, IEEE, Andreja Samčović, \\ Milan Milivojević, Goran Zajić, and Valery Starovoitov, Member, IEEE
}

\begin{abstract}
Luminance compression is often performed for high dynamic range images (still images and videos). A nonlinear tone mapping is applied for the compression in order to reproduce high dynamic range images using devices with a more limited (low) dynamic range. The images obtained after mapping may provide significant content differences in comparison to original data. This can be found for both optical and non-optical images. In this paper, we consider non-optical high dynamic range images, such as synthetic aperture radar images. Particularly, luminance compression may produce unwanted effects. Artificial objects found in an image and speckle noise may significantly affect the quality after tone mapping. In this paper, we consider several examples related to synthetic aperture radar images, as well as several global and a local luminance reduction method. The experimental analysis includes a comparison of several quality assessment methods.
\end{abstract}

Keywords - High dynamic range, synthetic aperture radar, luminance reduction, entropy, image quality.

Paper received April 6, 2018; revised May 29, accepted June 1, 2018. Date of publication July 31，2018. The associate editor coordinating the review of this manuscript and approving it for publication was Prof. Ljiljana Milić.

This paper is a revised and expanded version of the paper presented at the 25rd Telecommunications Forum TELFOR 2017 [19].

The research presented in this paper is partially funded by Ministry of Education, Science and Technological Development of the Republic of Serbia as a project TR32048, and Bilateral Project with Republic of Belarus on high dynamic image compression.

Ana Gavrovska is with the Department of Telecommunications, School of Electrical Engineering, University of Belgrade, Bulevar kralja Aleksandra 73, 11020 Belgrade, Serbia (e-mail: anaga777@gmail.com; anaga777@etf.rs).

Irini Reljin is with the Department of Telecommunications, School of Electrical Engineering, University of Belgrade, Bulevar kralja Aleksandra 73, 11020 Belgrade, Serbia (e-mail: irinitms@gmail.com; irini@etf.bg.ac.rs).

Andreja Samčović is with the Faculty of Transport and Traffic Engineering, Vojvode Stepe 305, 11000 Belgrade, Serbia (e-mail: andrej@sf.bg.ac.rs).

Milan Milivojević is with the Department of Telecommunications, School of Electrical Engineering, University of Belgrade, Bulevar kralja Aleksandra 73, 11020 Belgrade, Serbia (e-mail: msmilance@etf.rs).

Goran Zajić is with the ICT College of Vocational Studies in Belgrade, Zdravka Celara 16, 11020 Belgrade, Serbia (e-mail: gzajic@gmail.com).

Valery Starovoitov is with the United Institute of Informatics Problems, Minsk, Belarus (e-mail: valerystar@mail.ru).

\section{INTRODUCTION}

$\mathrm{T}$ HE ability of Human Visual System (HVS) to adjust to a wide range of light intensities and scene details have been a main concept in High Dynamic Range (HDR) imaging systems [1]. HDR image represents a wider range of luminance values in comparison to traditional/native low (standard) dynamic range (LDR) images. In a typical HDR imaging pipeline, HDR image can be reproduced using HDR or LDR display technologies. In the case of their reproduction on conventional LDR displays, luminance compression or tone-mapping is performed.

In the visual inspection of HDR and tone-mapped images there can be many similarities. Nevertheless, significant changes can also be found during the observation. For example, high intensity components found within an image after luminance compression may bring different information in comparison to an original HDR image [2].

The methods used for luminance reduction are applied to map the intensity levels from HDR into a lower dynamic range. Typically, the result after the reduction is an 8bit LDR image. There are many available methods for luminance compression. Most of them are performed for the reduction in the case of optical images [1]-[3].

Non-optical images may also refer to HDR data. Examples of non-optical HDR images can be found in biomedical data, like in computed tomography, SAR (synthetic aperture radar) data, etc. The methods for luminance reduction, as well as quality assessment, are valuable for this kind of images. After dynamic range reduction in non-optical images, the methods may affect specific details [4]-[5].

SAR images represent data acquired in remote sensing systems. This type of images is usually dark. A large number of pixel values can be found as in 8bit images. Nevertheless, a significant number of pixel intensities may be found outside the range $[0,255]$. Thus, some intensity values can be considered as saturated.

Considerable noise can also be found in non-optical images. In the presence of different noise statistics, the nonlinear mapping may affect the overall image quality. Image manipulation like image contrast enhancement may enhance the unwanted components. Speckle noise is found in SAR image, where it can be enhanced by luminance mapping. Moreover, regions of specific textures or high frequency components (edges) can be affected by inadequate mapping operators.

Valuable information in SAR images can be found both in low and high frequency bands in comparison to common 
optical images [6]. Specific image content statistics may produce a lower image quality after luminance compression. Also, artificial objects and specific materials like metal may provide extreme pixel values [7]-[10].

Thus, the high intensity changes found in pixels are treated by nonlinear methods, in order to introduce important intensity changes in the mapped image while preserving SAR image quality in low intensity segments. This is considered important in order to avoid any loss of valuable information.

Another important issue in non-optical images is how to evaluate the overall quality. There is a need for appropriate selection of non-reference metrics that can be used for quality assessment in the case of SAR images [9], [11].

In this paper, several standard global methods are considered for luminance range reduction. Moreover, some experiments are made from a singularity distribution standpoint. A mapping for SAR images is performed, where local regularity is considered. This paper is organized in five Sections. In Section II a brief description of HDR images and luminance reduction is given. Quality assessment is considered in Section III. The image analysis is described in Section IV. Section V presents some of the experimental results obtained by SAR image analysis, followed by discussion. Finally, in Section V conclusions are given.

\section{HIGH DYNAMIC RANGE NON-OPTICAL IMAGES}

\section{A. High dynamic range optical and non-optical images}

After capturing or generating an HDR image, it can be processed and visualized on an HDR monitor or tone mapped and reproduced as LDR image using traditional limited dynamic range display technologies. In the case when particular equipment for HDR acquisition does not exist, HDR image can be generated using a multiple exposure method [1]. Standard HDR pipeline is illustrated in Fig. 1. The obtained result after reducing dynamic range may convey different information in comparison to original data. There exist many different luminance compression methods, and their selection is crucial for a specific type of images.

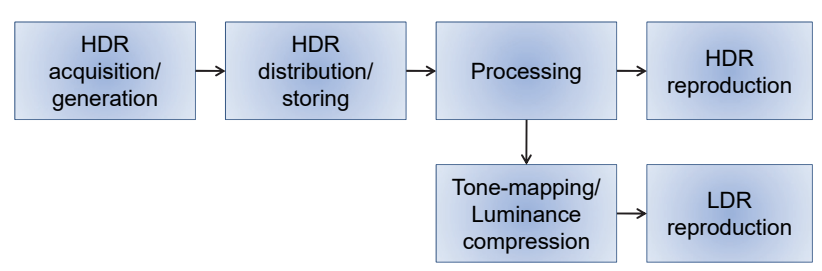

Fig. 1. Standard HDR pipeline and LDR reproduction.

Generally, visual imagery (a visible part of electromagnetic spectrum) is mostly analyzed in the literature [1]-[2]. Besides optical images, HDR can be found with non-optical sources. A typical example of HDR images is SAR. High intensities are found for specific materials and artificial objects. For non-optical SAR images luminance compression is also performed, where speckle noise can affect the selection of luminance compression method.

\section{B. Luminance compression}

For $m \times n$ HDR image $I(i, j), i=1, \ldots, m, j=1, . ., n$, compression of dynamic range is performed for reproduction in LDR domain. For luminance compression, values found in HDR images are often mapped into the normalized range $[0,1]$. Then, the range $[0,1]$ is linearly mapped to $[0,255]$, obtaining image in LDR domain $X(i, j)$.

Generally, for the purpose of luminance compression global and local methods can be used. The global methods are applied for each $(i, j)$ pixel in the same manner, where the local methods include information of pixel's neighborhood. Typically, nonlinear transforms are applied for luminance compression.

Luminance mapping affects both high and low intensities found in HDR, and they can be particularly sensitive during the reduction. For both high and low intensity values the luminance compression should provide a high quality result. In Fig. 2(a) a linearly mapped HDR SAR image is presented. The highest values are presented in Fig. 2(b).

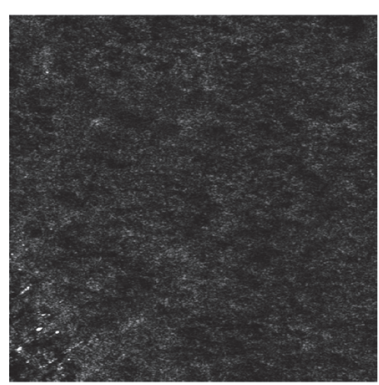

(a)

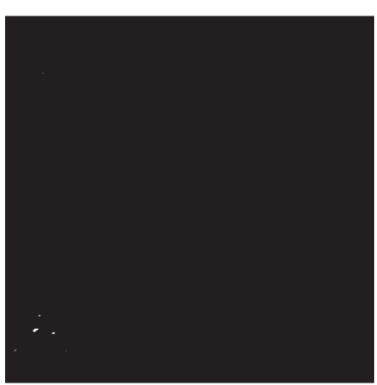

(b)
Fig. 2. (a) Linearly mapped SAR image and (b) its highest intensity values.

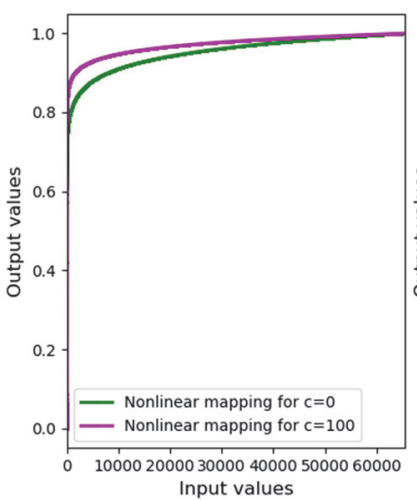

(a)

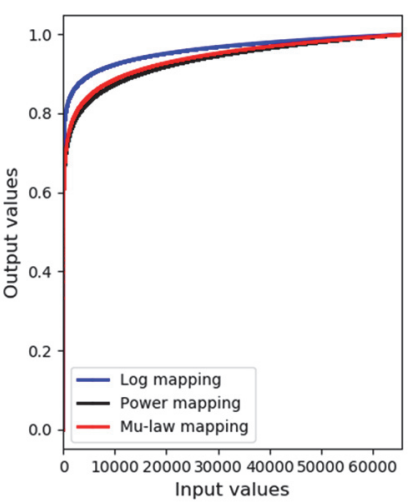

(b)
Fig. 3. Nonlinear mapping described by: (a) expression (1) for $c=0$ and $c=100$. (b) Standard global nonlinear mapping methods (log, power, mu-law).

The mapping is usually related to logarithm, exponential, power and similar functions. For example, a nonlinear mapping to perform the high dynamic range reduction [12], can be written as:

$$
X(i, j)=\operatorname{sgn}(I(i, j)-c)(|I(i, j)-c|)^{b},
$$

where sgn represents the sign function, and $(i, j)$ determines pixel position. In Fig.3(a) the mapping is presented.

Nonlinear mapping methods, as presented in Fig. 3, for 
luminance reduction are often defined as monotonically increasing or S-shaped [7]. Parameters such as $b$ and $c$ in (1) can be selected as constants for all pixels in a global method, or selected according to particular positions in a local method. In a local method, for $b=b(i, j)$ and $c=c(i, j)$, the values in LDR image $X$ depend on the neighborhood of pixel position $(i, j)$.

\section{QUALITY ASSESSMENT}

Performance evaluation of luminance compression should be performed in an automatic and objective manner. The subjective evaluation is not efficient, and it is timeconsuming. Development of independent quality assessment (IQA) models is particularly important for performance evaluation after luminance reduction. The comparison between images before and after mapping is not an easy task, and there are not so many metrics available for the comparison among images with different dynamic ranges [13]-[16].

Evaluation of a luminance reduction method can be performed: using a reference (FR - Full Reference), using partially a reference (PR - Partially Reference), and without using a reference (NR-Non-Reference). Targeting a result or using a reference is often not available in practical implementations. If a full reference image does not exist, there is a possibility to use some image attributes for PR approaches. NR quality assessment is believed to be particularly important for tone mapping. One should have in mind that after luminance reduction, it is usually not possible to have knowledge about the HDR information in LDR image content. This means that all the information from HDR image may not be contained in the mapped image.

Typical objective image quality assessment assumes the reference usage and the same dynamic range. Such assessments are not applicable for the HDR-LDR mapping comparison. The non-reference quality measures should be relatively efficient in estimating the obtained quality without significant computational loads. Dynamic range reduction methods can be also based on maximizing nonreference metrics [9]. There is a need for a numerical value that efficiently describes the overall quality of SAR and other non-optical images.

One of the methods for quality assessment in image and video engineering is a structure similarity (SSIM) index, which may overcome the assessment using measures, such as a mean squared error (MSE) or peak signal-to-noise ratio (PSNR). It is described as:

$$
\operatorname{SSIM}(A, B)=\frac{2 \mu_{A} \mu_{B}+C_{1}}{\mu_{A}^{2}+\mu_{B}^{2}+C_{1}} \cdot \frac{2 \sigma_{A B}+C_{2}}{\sigma_{A}^{2}+\sigma_{B}^{2}+C_{2}}
$$

where $\mu_{A}$ and $\mu_{B}$ represent mean intensity values, and $\sigma_{A}$ , $\sigma_{B}, \sigma_{A B}$ standard deviations of inputs $A, B$ and their crosscorrelation. As measures of structural similarity among the low and high dynamic range image SSIM and M-SSIM (modified SSIM) indexes are calculated [14]. Generally, SSIM includes perception-based information to describe the changes found among the two images.

One of the most relevant quality assessment methods for HDR optical images is Tone Mapped image Quality Index
(TMQI) [13]. TMQI combines multiscale fidelity and naturalness measure, and is often applied for evaluation of optical images with the compressed dynamic range. TMQI is motivated by SSIM structural fidelity measure with statistical evaluation of naturalness. If a new structural measure is described locally as:

$$
S_{l o c}(A, B)=\frac{2 \sigma_{A}^{\prime} \sigma_{B}^{\prime}+C_{1}}{\sigma_{A}^{\prime 2} \sigma_{B}^{\prime 2}+C_{1}} \cdot \frac{\sigma_{A B}+C_{2}}{\sigma_{A} \sigma_{B}+C_{2}}
$$

where $\sigma^{\prime}$ denotes a standard deviation after mapping, structural fidelity TMQI-S $(S)$ is described according to physiological experiments [13], where for each scale $l$, averaging is performed locally:

$$
S=\prod_{l=1}^{L} S_{l}^{\beta_{l}}, \quad S_{l}=\frac{1}{N_{l}} \sum_{i=1}^{N_{l}} S_{l o c}\left(A_{i}, B_{i}\right) .
$$

The statistical naturalness score TMQI-N $(N)$ can be described by modeling in the case of a large number of natural images using Gauss and Beta distributions [13]:

$$
\begin{gathered}
N=\frac{1}{\max \left(P_{m}, P_{d}\right)} P_{m} P_{d}, \\
P_{m}(m)=\frac{1}{\sqrt{2 \pi \sigma_{m}}} \exp \left[-\frac{m-\mu_{m}}{2 \sigma_{m}^{2}}\right], P_{d}(d)=\frac{(1-d)^{\beta_{d}-1} d^{\alpha_{d}-1}}{B\left(\alpha_{d}, \beta_{d}\right)}
\end{gathered}
$$

Finally, TMQI or TMQI-Q $(Q)$ gives the overall score of the obtained image in LDR domain:

$$
T M Q I(A, B)=k[S(A, B)]^{t_{1}}+(1-k)[N(A, B)]^{t_{2}}
$$

where $k, t_{1}, t_{2}$ are obtained experimentally [13].

\section{SimUlation}

In this paper, SAR images of size $998 \times 998$ are tested. The pixel intensities in each image belong to a range [0,65535]. Each of the tested images has neighboring pixels with large pixel intensities.

First, three global methods are applied to perform the high dynamic range reduction:

$$
\begin{gathered}
X_{1}(i, j)=\log (\max (a, I(i, j))), \\
X_{2}(i, j)=(I(i, j))^{b}, \\
X_{3}(i, j)=\operatorname{sgn}(I(i, j)) \log (1+\mu|I(i, j)|) / \log (1+\mu) .
\end{gathered}
$$

In the second part of the experiment, Hoelder exponents $\alpha$ are calculated. The Hoelder exponent is defined for a function $f$ on the interval as:

$$
|f(x)-f(y)| \leq k|x-y|^{\alpha},
$$

where $k$ is a constant. The coarse Hoelder exponent describes a signal or image locally using a measure $\mu$ (e.g. an input image based sum):

$$
\alpha=\lim _{\varepsilon \rightarrow 0} \log \mu(\varepsilon) / \log (\varepsilon),
$$

and can be calculated for each point. Thus, it enables to calculate the alpha matrix, named image $\mathrm{H}$ with values $\alpha(i, j)$ in the range $\left[\mathrm{H}_{\min }, \mathrm{H}_{\max }\right]$ described by extreme alpha values. Spectrum based on the values presents a distribution like representation of the calculated exponents. For the experimental setup, the exponents are considered to be useful to describe the local changes found in SAR image. Inverse histogram method is applied for the spectrum calculation and specific point detection within alpha range [17]. In order to reduce the noise effect which may affect image naturalness in LDR domain, median filtering is applied to preprocess an image. This is applied only for the points with the highest alpha values (for $\mathrm{H}>\mathrm{T}_{0}$ ). The 
singularities related to noise and similar behavior are roughly described as points in alpha image where $\mathrm{H}>\mathrm{T}_{0}>\mathrm{T}$, and these points are changed to value $\mathrm{T}$ in $\mathrm{H}$ matrix (here $\mathrm{T}=1$ ). After the preprocessing, a power transform is applied as:

$$
X_{4}(i, j)=I_{\log , \text { norm }}^{b_{\text {nom }}(i, j)}(i, j),
$$

where $I_{\log }$ and $b$ are selected as the result of logarithm mapping and the calculation of alpha values found in $\mathrm{H}$ matrix.

The obtained results are normalized for each input:

$$
\text { output }(i, j)=\frac{\text { input }(i, j)-\min (\text { input }(i, j))}{\max (\text { input }(i, j))-\min (\text { input }(i, j))}
$$

and linearly mapped to $[0,255]$. Several measures are used for quality assessment, such as TMQI and entropy. Entropy is calculated to describe the average of information [2].

\section{EXPERIMENTAL RESULTS}

The representation of $200 \times 200$ segments linearly mapped is illustrated in Fig. 4. Each of the segments contains objects with extreme intensity pixels, represented as saturated (black and white) pixels. The white connected regions represented in an 8 bit unsigned integer format can be found in both rural and urban areas. In Fig. 5 the significant intensity changes found in pixels may differentiate from the positions of extreme values, as in Fig. 2.
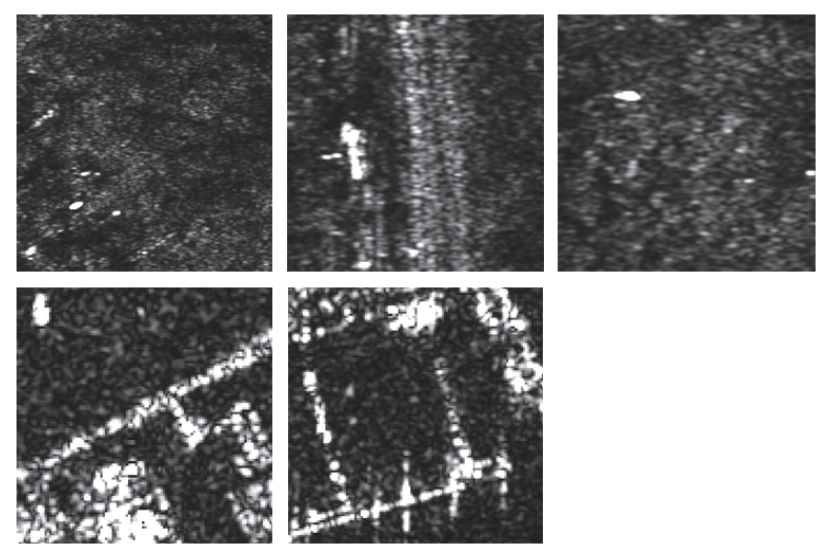

Fig. 4. High intensity objects found in different parts of SAR images (linearly mapped).

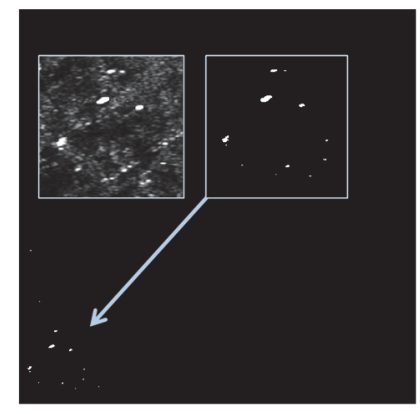

Fig. 5. The significant intensity changes after mapping.

In the lower part of Fig. 4, examples characterized by a larger number of high intensity values, probably belonging to more urban areas, are presented.

First three global methods (7)-(9) are tested. These tests include the standard mapping denoted as: log, power and mu-law, where mu-law gave satisfying results among the three abovementioned methods. Similarly is obtained in [7].

The dynamic range reduction is also performed using local regularity calculation in order to identify the points that may affect the overall quality. The fourth test is based on the analysis of the alpha values calculated from an image. As an example, the obtained alpha values generating the $\mathrm{H}$ matrix is shown in Fig. 6. A larger spectrum width is found in the examples like in the lower part of Fig. 4.

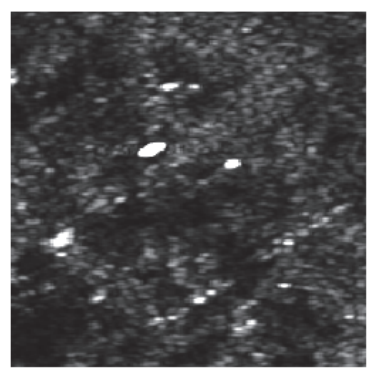

Fig. 6. An image part of uint8 representation and its $\mathrm{H}$ matrix.

The points with alpha values less than 0.87 give similar representation as in Fig. 5, where the significant intensity changes are noticeable. The higher alpha values (above 1.2) seem to be related to noise, which are used in the preprocessing step. Characteristic pixels can be selected, as presented in Fig. 7.

In Fig. 7(b) it is shown that relevant pixels with high intensity values can be segmented. In the tested images the lower intensities are related to areas like: shadows, river, sea regions, etc.

\section{A. Luminance range reduction results}

The results of four tests are shown in Table 1. The results are presented for five images, where the fourth and fifth image have a significant number of high intensity values. In Fig. 8 some parts of HDR reduction results in test 4 are presented.
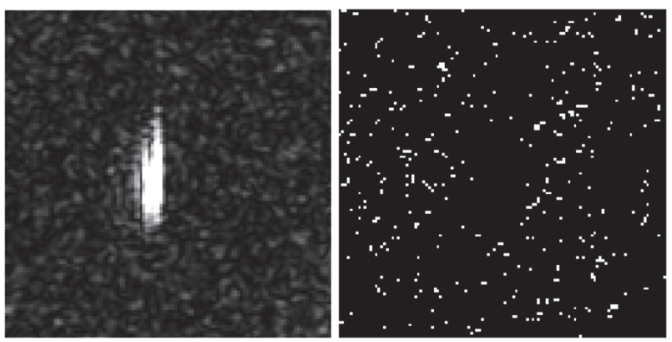

(a)
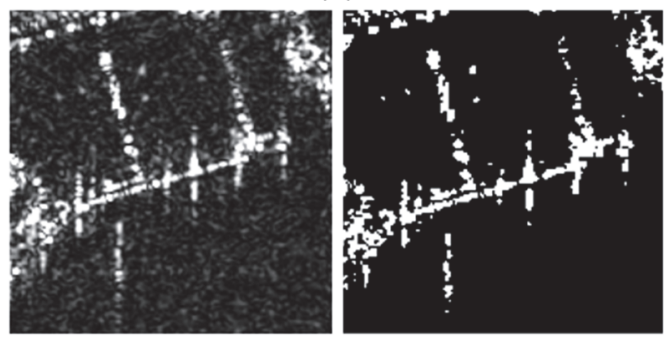

(b)

Fig. 7. (a) Noise effect for the highest alpha values (b) Segmented relevant pixels. 
TABle 1: Performance EVAluation

\begin{tabular}{|c|c|c|c|c|c|c|}
\hline $\begin{array}{c}\text { Sour- } \\
\text { ce }\end{array}$ & Entropy & SSIM & $\begin{array}{c}\text { M- } \\
\text { SSIM }\end{array}$ & $\begin{array}{c}\text { TMQI- } \\
\text { N }\end{array}$ & $\begin{array}{c}\text { TMQI- } \\
\text { S }\end{array}$ & $\begin{array}{c}\text { TMQI- } \\
\text { Q }\end{array}$ \\
\hline \multicolumn{7}{|c|}{ Test1-log } \\
\hline S_01 & 5.801 & 0.796 & 0.779 & 0.388 & 0.983 & 0.899 \\
\hline S_02 & 6.139 & 0.655 & 0.637 & 0.833 & 0.980 & 0.971 \\
\hline S_03 & 5.876 & 0.687 & 0.668 & 0.780 & 0.978 & 0.963 \\
\hline S_04 & 5.287 & 0.149 & 0.187 & 0.011 & 0.867 & 0.775 \\
\hline S_05 & 5.477 & 0.173 & 0.196 & 0.023 & 0.814 & 0.766 \\
\hline \multicolumn{7}{|c|}{ Test2-power } \\
\hline S_01 & 5.770 & 0.841 & 0.826 & 0.232 & 0.984 & 0.868 \\
\hline S_02 & 6.144 & 0.699 & 0.681 & 0.665 & 0.981 & 0.945 \\
\hline S_03 & 5.870 & 0.733 & 0.715 & 0.593 & 0.979 & 0.933 \\
\hline S_04 & 5.651 & 0.202 & 0.252 & 0.148 & 0.898 & 0.827 \\
\hline S_05 & 5.784 & 0.229 & 0.263 & 0.252 & 0.853 & 0.838 \\
\hline \multicolumn{7}{|c|}{ Test3 - mu-law } \\
\hline S_01 & 5.800 & 0.797 & 0.779 & 0.387 & 0.983 & 0.899 \\
\hline S_02 & 6.136 & 0.655 & 0.637 & 0.832 & 0.980 & 0.971 \\
\hline S_03 & 5.874 & 0.687 & 0.668 & 0.779 & 0.978 & 0.962 \\
\hline S_04 & 5.880 & 0.234 & 0.293 & 0.207 & 0.897 & 0.840 \\
\hline S_05 & 6.044 & 0.259 & 0.301 & 0.351 & 0.863 & 0.861 \\
\hline \multicolumn{7}{|c|}{ Test4 - local } \\
\hline S_01 & 6.056 & 0.802 & 0.789 & 0.512 & 0.993 & 0.923 \\
\hline S_02 & 6.355 & 0.667 & 0.651 & 0.961 & 0.989 & 0.992 \\
\hline S_03 & 6.087 & 0.690 & 0.675 & 0.923 & 0.990 & 0.987 \\
\hline S_04 & 6.667 & 0.448 & 0.537 & 0.873 & 0.940 & 0.967 \\
\hline S_05 & 6.606 & 0.467 & 0.537 & 0.889 & 0.918 & 0.964 \\
\hline
\end{tabular}

Even though the overall quality evaluated by TMQI-Q is relatively high in each test, it can be seen that for the tests $1-3$, naturalness can be relatively low (TMQI-N). Similarly, low values are obtained for SSIM and M-SSIM measures.

By analyzing local regularity in the fourth test, higher values for both TMQI-N and TMQI-S are obtained in comparison to the previous tests. Test 4 gave also higher values for SSIM. The highest overall TMQI-Q values are obtained in test 4 . Some of the interesting values in the table are bolded. Moreover, the highest entropy values are obtained in the last test.

\section{B. Quality assessment relation}

After analyzing the dataset consisting of the above twenty examples, images with high and low entropy values can be noticed, as presented in Fig. 9. The performance evaluation showed different behavior for TMQI and entropy in comparison to HDR entropy values. In Fig. 9 the obtained results in test 4 are presented. Entropy for the mapped images is normalized. The analysis based on entropy showed satisfying results. Linear regression provided a mean squared error (MSE) of 0.055 [18].
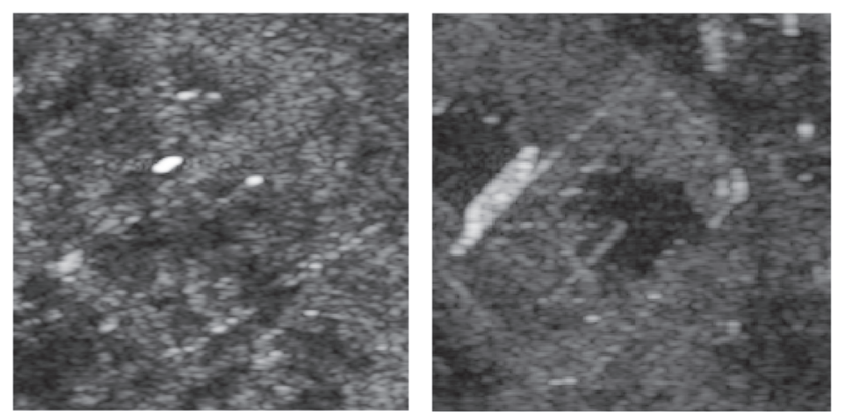

Fig. 8. High dynamic range reduction result.

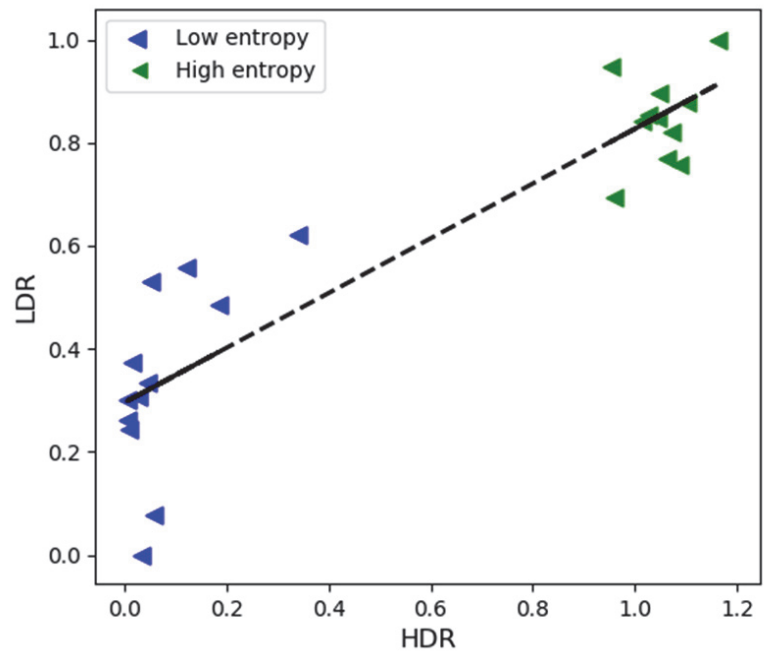

Fig. 9. Entropy analysis and linear regression.

In Fig. 10 TMQI-Q dependence on structural fidelity and naturalness is presented. For test 4 it can be seen that a high correlation with naturalness has been obtained (MSE= 0.084). Nevertheless, it also showed there is a satisfying correlation with SSIM. An adequate balance between structural fidelity and naturalness should be obtained.

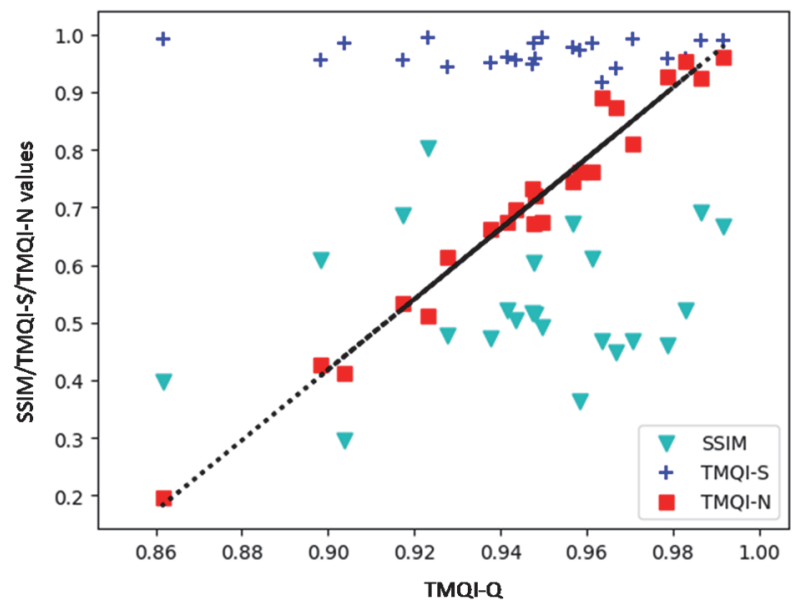

Fig. 10. TMQI-Q dependence on structural fidelity and naturalness, with the ordinate corresponding to the calculated values of SSIM, TMQI-S and TMQI-N.

\section{CONCLUSION}

Some of the experimental results for luminance range reduction in high dynamic range images are presented in this paper. Namely, the compression of high dynamic range may affect the information contained in the original HDR image. The compression for non-optical images has not been analyzed as much as for the optical images. SAR images as non-optical images can represent HDR data. The overall quality can be affected after tone mapping since they are characterized by speckle noise and often contain high intensity values due to artificial objects and specific materials. Both good structure fidelity and naturalness should be obtained in LDR domain. Our results show that investigating the local changes may be helpful in providing 
good overall quality regardless of the input (images representing rural and urban areas). They show that it is possible to increase both naturalness and structure fidelity, where entropy evaluation has an important role. Further work can be oriented towards additional examinations of the local regularities found in this type of images. Moreover, both local and global quality assessments are valuable for specific non-optical HDR images, like SAR, and should be further investigated.

\section{REFERENCES}

[1] F. Banterle, A. Artusi, K. Debattista, and A. Chalmers: ,Advanced high dynamic range imaging: theory and practice", CRC Press, 2011.

[2] A. Gavrovska, I. Reljin, A. Samčović, V. Starovoitov, and M. Milivojević, "Comments on Human Visual Attention in High Dynamic Range Images"; Proceedings of the 4th International Conference on Electrical, Electronic and Computing Engineering IcETRAN 2017, EK(I)2-1- 4, Kladovo, Serbia, June 5-8, 2017.

[3] A. Gavrovska, A. Samčović, "Mogućnosti unapređenja kvaliteta slike i videa viokog dinamičkog opsega," 34. simpozijum o novim tehnologijama u poštanskom i telekomunikacionom saobraćaju, PosTel2016, Belgrade, Nov. 2016, pp. 265-274.

[4] M. Lambers, H. Nies, and A. Kolb, "Interactive dynamic range reduction for SAR images", Geoscience and Remote Sensing Letters, vol. 5, no. 3, pp. 507-511, 2008.

[5] M. Lambers and A. Kolb, "Adaptive dynamic range reduction for SAR images". In Proc. 7th European Conference on Synthetic Aperture Radar (EUSAR), vol. 3, pp. 371-374, 2008.

[6] G. Arslan and M. Valliappan, "Literature Survey on Synthetic Aperture Radar (SAR) Image Compression", EE381K Multidimensional Signal Processing, 1998.

[7] V. Starovoitov, "High Dynamic Range SAR Image Compression for Visualization ", pp. 218-222, 2016.

[8] J. B. Zhang, C. Wang, H. Zhang and F. Wu, "Adaptive intensity compression for high dynamic SAR image", Proceedings of 10th
European Conference on Synthetic Aperture Radar (EUSAR), pp. 497-500, 2014

[9] B. Zhang, C. Wang, H. Zhang and F. Wu, "An adaptive two-scale enhancement method to visualize man-made objects in very high resolution SAR images." Remote Sensing Letters 6, no. 9, pp. 725$734,2015$.

[10] S. Hisanaga, K. Wakimoto and K. Okamura, "Tone mapping and blending method to improve SAR image visibility", IAENG International Journal of Computer Science, 38(3), pp.289-294, 2011.

[11] A. Gavrovska, A. Samčović, "Procena smanjenja dinamičkog opsega," 35. simpozijum o novim tehnologijama u poštanskom $i$ telekomunikacionom saobraćaju, PosTel2017, Belgrade, Dec. 2017, pp. 221-230.

[12] J. Ok and C. Lee, "HDR tone mapping algorithm based on difference compression with adaptive reference values," Journal of Visual Communication and Image Representation, 43, pp.61-76, 2017.

[13] H. Yeganeh and Z. Wang, "Objective Quality Assessment of Tone Mapped Images," IEEE Transactions on Image Processing, vol. 22(2), pp.657-667, Feb. 2013

[14] Z. Wang, A. C. Bovik, H. R. Sheikh and E. P. Simoncelli, "Image quality assessment: From error visibility to structural similarity," IEEE Transactions on Image Processing, vol. 13, no. 4, pp. 600-612, Apr. 2004.

[15] P. Hanhart, M. Bernardo, P. Korshunov, M. Pereira, A. Pinheiro, T. Ebrahimi: „HDR image compresion: a new challenge for objective quality metrics“, Expert Systems with Applications, Vol. 42, No. 9, pp 4177-4195, 2015

[16] Y. Fu and S. Wang, "A no reference image quality assessment metric based on visual perception," Algorithms, 9(4), p.87, 2016.

[17] A. Pavlović, A. Gavrovska, and I. Reljin, "Multifractal spectrum of the images obtained by copy move method," Proceedings of the 4th International Conference on Electrical, Electronic and Computing Engineering IcETRAN 2017, pp. EKI1-1- 4, Kladovo, Serbia, June 5-8, 2017.

[18] Scikit-learn, http://scikit-learn.org/stable/ (last accessed 04.03.2018.).

[19] A. Gavrovska, I. Reljin, A. Samčović, M. Milivojevic, G. Zajić and V. Starovoitov, "On luminance reduction in high dynamic range synthetic aperture radar images," 2017 25th Telecommunication Forum (TELFOR), Belgrade, 2017, pp. 1-4. 